\title{
Percutaneous Thoracic Intervertebral Disc Nucleoplasty: Technical Notes from 3 Patients with Painful Thoracic Disc Herniations
}

\author{
Nicholas H. L. Chua ${ }^{1,2}$, Ismail Gültuna ${ }^{3}$, Patricia Riezebos², Tjemme Beems ${ }^{4,5}$, Kris C. Vissers ${ }^{2}$ \\ 'Department of Anesthesiology and Pain Management, Tan Tock Seng Hospital, Singapore \\ ${ }^{2}$ Department of Anesthesiology, Pain and Palliative Care, \\ Radboud University Nijmegen Medical Centre, Nijmegen, The Netherlands \\ ${ }^{3}$ Department of Anesthesiology and Pain Management, Albert Schweitzer Hospital, Sliedrecht, The Netherlands \\ ${ }^{4}$ Department of Neurosurgery, Radboud University Nijmegen Medical Centre, Nijmegen, The Netherlands \\ ${ }^{5}$ TWIN Institute of Neuromodulation, Nijmegen, The Netherlands
}

\begin{abstract}
Symptomatic thoracic disc herniation is an uncommon condition and early surgical approaches were associated with significant morbidity and even mortality. We are the first to describe the technique of percutaneous thoracic nucleoplasty in three patients with severe radicular pain due to thoracic disc herniation. Two of the patients experienced more than $75 \%$ pain relief and one patient experienced more than $50 \%$ pain relief. Post-procedural pain relief was maintained up to an average of 10 months after nucleoplasty. One patient with preoperative neurological signs improved postoperatively. There were no reported complications in all three patients. In view of the reduced morbidity and shorter operating time, thoracic interver tebral disc nucleoplasty can be considered in patients with pain due to thoracic disc herniation, with no calcification of the herniated disc, and in patients who may be otherwise be unfit for conventional surgery.
\end{abstract}

Key Words: Thoracic intervertebral disc herniation, Thoracic nucleoplasty, Percutaneous thoracic disc cannulation, Minimally invasive procedures

\section{Introduction}

Symptomatic thoracic disc herniation is an uncommon condition, accounting for approximately five of every 1,000 disc herniations encountered in the clinical setting [1]. Majority of thoracic disc herniations are asymptomatic [2], with radicular chest pain being the most common presenting complaint. Few patients require invasive treatments and most conservatively treated patients eventually returned to the prior level of activity [3].

Earlier cases that require surgical intervention required a dorsal surgical approach which was associated with significant complications of irreversible paraplegia and operative mortalities approaching 10\% [4]. Current surgical techniques have demonstrated significant improvements in the postoperative critical care requirements and discharge times. However, these techniques all require general anesthesia and in many instances, one-lung ventilation. Furthermore, added neurological complications, pneumothorax, blood loss and surgical wound infection are all inherent surgical complications. There is also a significant risk of intercostal neuralgia as reported by Rosenthal and Dickman [5]: $16 \%$ in thoracoscopy, $20 \%$ when via costotransversectomy

Received Jul 2, 2010; 1st Revised Jul 29, 2010; Accepted Aug 26, 2010

Corresponding author: Nicholas H. L. Chua, MBBS, MMed, FIPP, DAAPM

Department of Anaesthesiology, Tan Tock Seng Hospital, 11 Jalan Tan Tock Seng, Singapore 308433

Tel: +65-6357-7771, Fax:+65-6357-7772, E-mail: nicholaschua143@gmail.com 
and $50 \%$ in patients who had undergone a thoracotomy.

Nucleoplasty is a minimally invasive procedure which uses radiofrequency energy to remove nuclear material and create small channels within the disc [6]. Having been described for cervical and lumbar disc prolapse, the use of nucleoplasty had not previously been described for thoracic levels. The partial removal of the nucleus pulposus in contained thoracic disc prolapses may similarly decompress herniated discs and relieve pressure on nerve roots [7]. In addition to surgical risk reduction, the advantages of a percutaneous procedure for thoracic disc pathologies are shorter operative times and improved post-operative recovery process. The cannulation of thoracic intervertebral discs however requires utmost precision [8] as the puncture of the pleura, dura, spinal cord are all potential hazards [9]. Beam hardening from the overlying ribs and a narrower intervertebral space provide added challenges to thoracic disc cannulation $[8,9]$. We describe the use of a percutaneous thoracic nucleoplasty technique for the first time, in 3 consecutive patients with severe radicular pain due to thoracic disc herniation who were otherwise unfit for surgery from Jan 2008 to September 2009.

\section{Technical Notes}

Three patients presented with varying symptoms of severe thoracic radicular pain. The duration, location and character of the pain are summarised in Table 1. All three patients had preoperative thoracic magneric resonance imaging (MRI) scans but only the first patient underwent a postoperative MRI. All 3 patients had their procedures performed with ArthroCare Perc DLG SpineWand ${ }^{\circledR}$ (ArthroCare, Austin, TX, USA) and a standard 17 gauge $\times 8^{\prime \prime}$ Crawford needle, with the ArthroCare System 2000 Controller. The same physician performed all procedures in a prone position using a uniportal approach under fluoroscopic guidance, entering the disc from the side of predominant pain. The patient is positioned in a prone position with a small pillow under his chest. The fluoroscope is first tilted in a cephalad-caudal direction such that both endplates are levelled and the disc is "opened up." An oblique view of 20-25 degrees is then obtained such that the interpedicular line lies almost to the middle of the vertebral bodies in this plane. It is important to obtain the required amount of oblique view, as a less than adequate rotation will result in too lateral a placement of the needle.

Table 1. Summary of patient information including pain locations, neurological grading, previous procedures, thoracic nucleoplasty details and post-procedure complications

\begin{tabular}{|c|c|c|c|}
\hline & Patient 1 & Patient 2 & Patient 3 \\
\hline \multicolumn{4}{|l|}{ Pre-operative } \\
\hline Age (yr) & 50 & 52 & 78 \\
\hline Gender & Female & Male & Female \\
\hline Location of pain & $\begin{array}{l}\text { Right T7 and left } \\
\text { T6 dermatomes }\end{array}$ & $\begin{array}{l}\text { Left T12 dermatome } \\
\text { with paraesthesia }\end{array}$ & Right L1 dermatome \\
\hline Duration of pain (thoracic) in years & 3 & 0.25 & 8 \\
\hline Previous procedures & T7 DRG diagnostic block & Nil & Hemiarthroplasty of right hip \\
\hline Levels of thoracic disc herniation on & $\mathrm{T} 6 / 7$ & $\mathrm{~T} 11 / 12$ & $\mathrm{~T} 12 / \mathrm{L} 1$ \\
\hline MRI & $\mathrm{T} 7 / 8$ & & \\
\hline Frankel grade & $\mathrm{E}$ & $\mathrm{D}$ & $\mathrm{E}$ \\
\hline \multicolumn{4}{|l|}{ Intra-operative } \\
\hline Nucleoplasty performed at levels & $\mathrm{T} 6 / 7$ & $\mathrm{~T} 11 / 12$ & $\mathrm{~T} 12 / \mathrm{L} 1$ \\
\hline & $\mathrm{T} 7 / 8$ & & \\
\hline Duration of procedure (min) & 45 & 35 & 40 \\
\hline Duration of hospitalisation (night) & 1 & 1 & 1 \\
\hline \multicolumn{4}{|l|}{ Post-operative } \\
\hline Pain relief at 6 months & $>75 \%$ & $>75 \%$ & $>50 \%$ \\
\hline Duration of pain relief (months) & 13 & 12 & 6 \\
\hline Post-procedure MRI & Yes & No & No \\
\hline Volumetric reduction on MRI & Yes & - & - \\
\hline Complications & Nil & Nil & Nil \\
\hline
\end{tabular}

DRG: Dorsal root ganglion, MRI: Magnetic resonance imaging. 
After aseptic preparation of the skin, the needle is directed in a tunneled view (Fig. 1), just lateral to the interpedicular line, keeping the needle tip medial to the costovertebral joint [9]. This is important to prevent the pleura from being penetrated. At times, the pleura may be breached even if such an approach is performed. The bent-needle technique is hence ideal. Two factors work upon the bent needle and act synergistically with the trocar to impart a medial movement on the inner needle: (1) The bend, which is oriented toward the disc; and (2) the bevel, which faces laterally, facilitate the tip to move medially upon purchasing soft tissue such as the annulus [8]. The bent-needle technique thus allows a more medial entry, just lateral to the interpedicular line and enables the needle tip to be steered laterally around the superior articular process into the herniated thoracic disc. We bend the needle approximately 1 inch from the tip with our thumbnail tangentially along the needle shaft (with the index finger under the needle) so the bevel faces away from the direction of the bend, with the trocar still engaged. The key to a good "bend" is having a smooth turn and tip in the same linear plane as the needle shaft. Care has to be taken then to ensure that the trocar can be retracted without

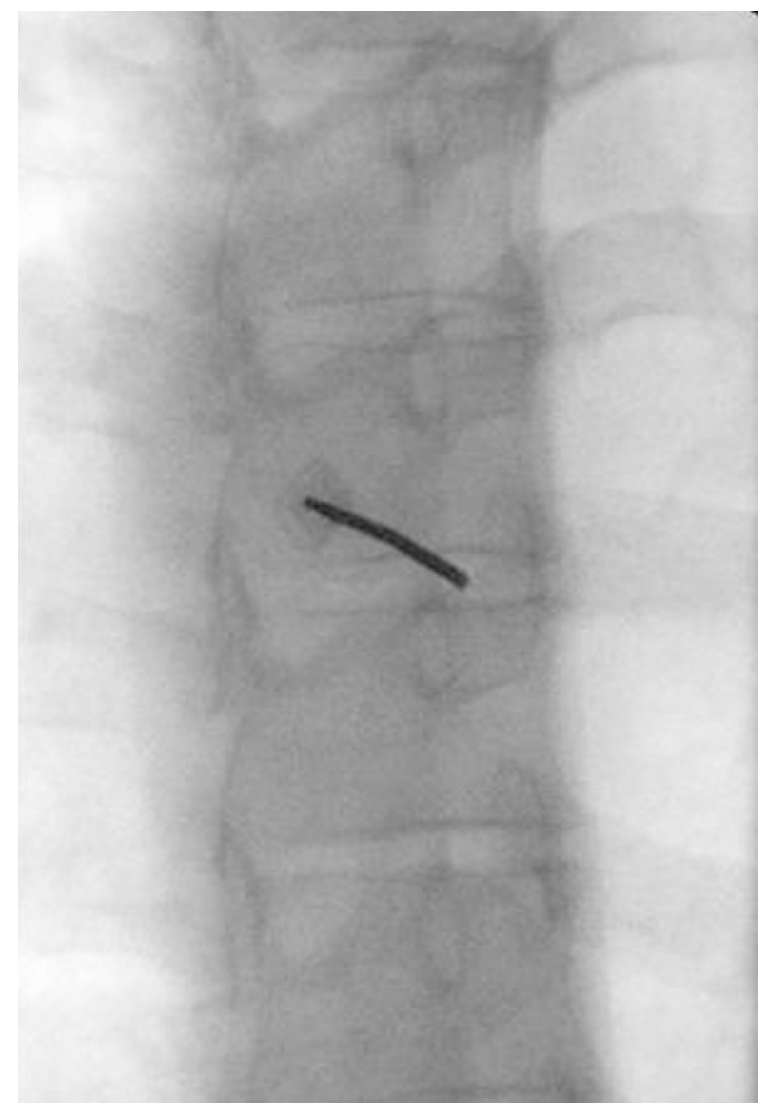

Fig. 1. Oblique entry view of introducer needle at T6-7 intervertebral disc. much resistance. This is followed by the insertion of the Perc-DLG tissue ablation and coagulation SpineWand ${ }^{\circledR}$ to verify the ease of its insertion and withdrawn without weakening or fracturing the tip.

Upon contact with the intervertebral disc, the annulus will provide initial resistance and will feel "gritty" as the needle passes through it. In the thoracic intervertebral disc, this will be followed by a distinct loss of resistance as the needle enters the nucleus pulposus. The needle tip should be placed in the transitional zone between the annulus and the nucleus. This would correspond fluoroscopically to the posterior one-third of the disc space on the lateral view. The SpineWand ${ }^{\circledast}$ is then inserted and advanced until the tip of the wand is approximately $5 \mathrm{~mm}$ beyond the tip of the cannula, assuring that the active portion of the wand was beyond the inner layer of the annulus and in the nucleus. The depth is then confirmed in both the postero-anterior and lateral fluoroscopic views (Fig. 2). The wand is then advanced until it comes into contact with the annulus on the opposite side, which will offer some resistance. The depthstop marker on the shaft of the Perc-DLG SpineWand ${ }^{\circledR}$ is advanced close to the needle hub to designate the distal channeling limit. The process of decompression involves advancing the wand, in ablation mode, at a speed of 0.5 $\mathrm{cm} / \mathrm{sec}$ and, similarly, retraction of the wand was performed in coagulation mode at a speed of $0.5 \mathrm{~cm} / \mathrm{sec}$. A total of six channels is created at the twelve, two, four, six, eight, and ten o'clock positions.

The first two patients experienced more than $75 \%$ pain relief and patient 3 experienced more than 50\% pain relief. Their pain relief was sustained up to the time of the writing of this manuscript with an average of 10 months of postprocedure relief. A thoracic MRI was repeated for patient 1, one month after the procedure which showed a near complete resolution of the T6/7 and T7/8 disc prolapse (Figs. 3

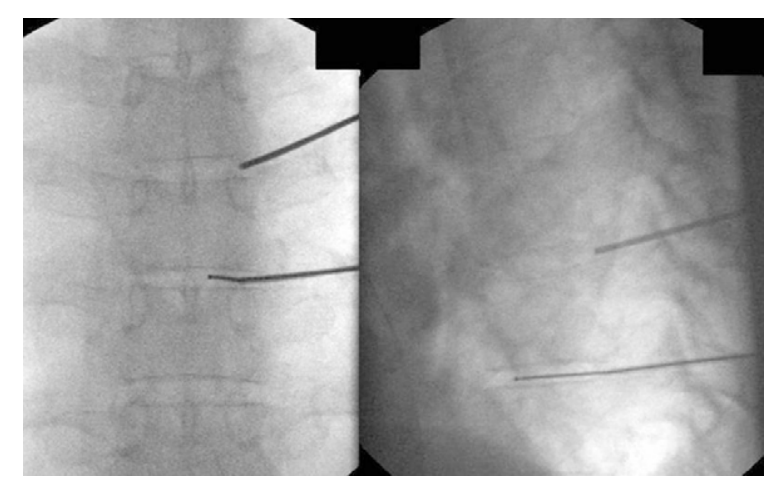

Fig. 2. Antero-posterior and lateral views of T6-7 and T78 nucleoplasty. 


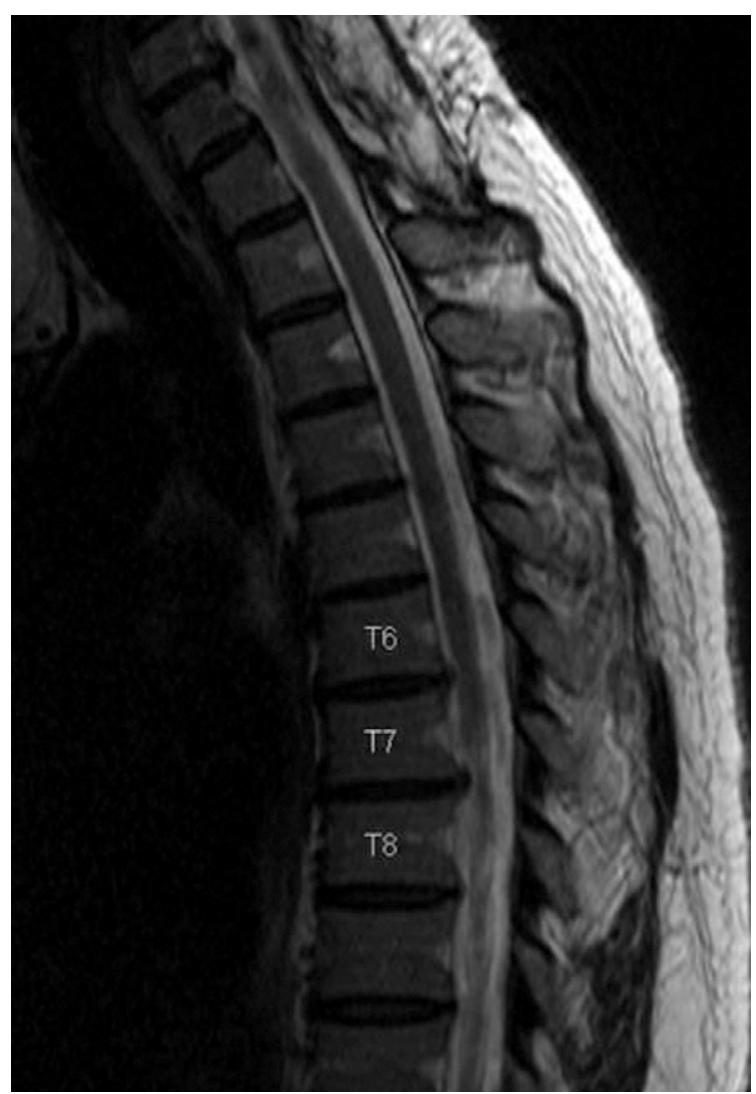

Fig. 3. Pre-procedure magnetic resonance imaging of patient 1.

and 4). However, pre and post-procedural MRI slices are not identical as they were performed in two different hospitals). Nucleoplasty of T11/12 was performed in patient 2 and the radicular left flank pain had completely resolved after the procedure along with the T11 dermatomal parasthesia. Patient 3 had incidental lower limb radicular symptoms which were not related to the T12/L1 disc prolapse. The pain over her right hip had resolved almost completely but lower limb symptoms remained. There were no reported post-operative neurological complications in all three patients.

\section{Discussion}

In their review of 280 casers, Arce and Dohrman [10] found an incidence of $75 \%$ of thoracic disc herniations below $\mathrm{T} 8$, with a peak incidence of $28 \%$ at $\mathrm{T} 11 / 12$. The reasons for this are due possibly to their anatomical characteristics, being thinner anteriorly and thicker posteriorly, in addition to the increased mobility in the lower segments [3]. Symptomatic herniated thoracic discs accounted for 0.25 to $0.75 \%$ of all disc ruptures and tend to present in the third to

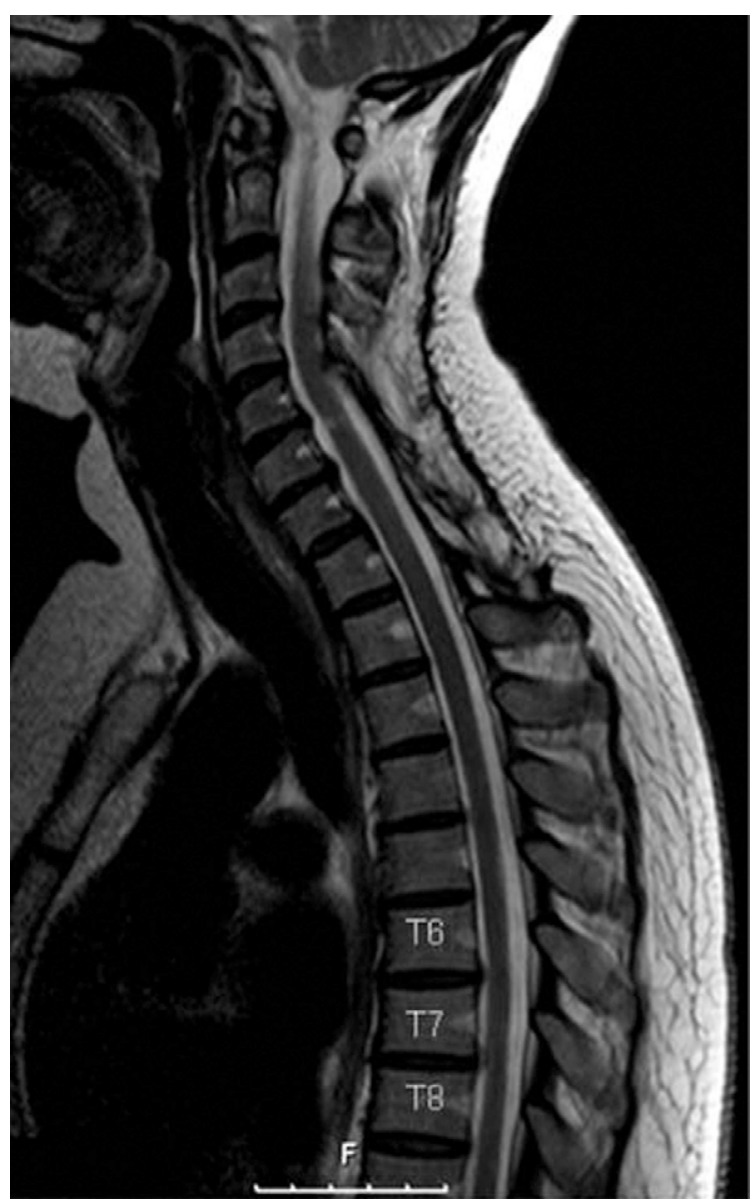

Fig. 4. Post-procedure magnetic resonance imaging of patient 1.

fifth decade of life, with a peak occurrence in the fourth decade $[3,10]$.

Thoracic intervertebral discs have a well-defined nucleus pulposus, but are smaller in volumetric dimension than the lumbar disc nucleus. The nucleus pulposus are more centrally located, surrounded by a dense fibroelastic annulus fibrosus. The volumetric reduction in the thoracic nucleus pulposus, may however result in a greater degree of neural decompression due possibly to the elliptical to circular shape of thoracic discs and its relative horizontal orientation.

All three of our patients have small disc herniations with pain as their main symptom. Patients with large herniations of more than $20 \%$ or calcified herniations are unlikely to benefit from such a procedure. Disc consistency is thus an important parameter influencing treatment. For the technique of thoracic disc nucleoplasty, we recommend additional computed tomography imaging to further delineate the extent of calcification, especially when this is suspected from conventional radiographic films or T1-weighted MR 
images. Furthermore, we are not advocating this as a procedure to replace essential function-salvaging surgery for neurological compromise but rather as a viable option in view of its reduced morbidity in patients with mainly pain as their chief complaint, with small herniations or for patients who are otherwise unfit for conventional surgery.

\section{REFERENCES}

1. Burke TG, Caputy AJ. Treatment of thoracic disc herniation: evolution toward the minimally invasive thoracoscopic technique. Neurosurg Focus 2000;9:e9.

2. Wood KB, Garvey TA, Gundry C, Heithoff KB. Magnetic resonance imaging of the thoracic spine: evaluation of asymptomatic individuals. J Bone Joint Surg Am 1995;77:1631-8.

3. Brown CW, Deffer PA Jr, Akmakjian J, Donaldson DH, Brugman JL. The natural history of thoracic disc hernia- tion. Spine (Phila Pa 1976) 1992;17(6 Suppl):S97-102.

4. Fessler RG, Sturgill M. Review: complications of surgery for thoracic disc disease. Surg Neurol 1998;49:609-18.

5. Rosenthal D, Dickman CA. Thoracoscopic microsurgical excision of herniated thoracic discs. J Neurosurg 1998;89:224-35.

6. Chen YC, Lee SH, Chen D. Intradiscal pressure study of percutaneous disc decompression with nucleoplasty in human cadavers. Spine (Phila Pa 1976) 2003;28:661-5.

7. Sharps LS, Isaac Z. Percutaneous disc decompression using nucleoplasty. Pain Physician 2002;5:121-6.

8. Fortin JD. Precision diagnostic disc injections. Pain Physician 2000;3:271-88.

9. Schellhas KP, Pollei SR, Dorwart RH. Thoracic discography: a safe and reliable technique. Spine (Phila $\mathrm{Pa}$ 1976) 1994;19:2103-9.

10. Arce CA, Dohrmann GJ. Herniated thoracic disks. Neurol Clin 1985;3:383-92. 